Schouten, J. F. and Ornstein, L. S. (1939).-Jl. Opt. Soc. Amer., Vol. XXIX, p. 168.

Sharplex, F. W. (1936).-Brit. Jl. Ophthal., Vol. XX, p. 129.

SHLAER, S. (1937).-Jl. Gen. Physiol., Vol XXI, p. 165.

TANSLEY, K. (1931).-Jl. Physiol., Vol LXXI, p. 442.

(1933).-Proc. Roy, Soc. B., Vol. CXIV, p. 79.

Trendelenburg, W. (1904).-Zeitsch Psychol. Physiol. Sinnesorg., Vol. XXXVII, p. 1.

WaLd, G. (1936).-Jl.gen. Physiol., Vol. XIX, p. 781.

(1938).-Jl. gen. Physiol., Vol. XXI. p. 795.

and Clar K, A. B. (1937).-Il. gen. Physiol., Vol. XXI, p. 93.

Wright, W. D. (1935).-Jl. Physiol., Vol. LXXXIII, p. 466.

- (1936),-Jl. Physiol., Vol. LXXXVII, p. 23.

(1937).-Proc. Roy. Soc. B., Vol. CXXII, p. 220.

ZEWI, M. (1939).-Acta Soc. scient. Fenn, Nova. Series B, II, No. 4.

\title{
SPHERO-CYLINDRICAL CONTACT LENSES- A PRELIMINARY NOTE
}

\author{
BY
F. A. Williamson-Noble, Josef Dallos and IDA MANN

Some two years ago, one of us was examining a patient who had been fitted with contact lenses and was disappointed to find that visual acuity with the contact lens was less good than with ordinary glasses. The spherical element was checked by the duochrome test and appeared to be right, so retinoscopy was performed and it was discovered that there was some residual astigmatism. When the correcting cylinder was placed in a trial frame in front of the eye wearing the contact lens an immediate improvement in visual acuity became manifest. It was then suggested that an endeavour should be made to grind the cylindrical correction on to the contact lens, but this could not be done with the machinery available at that time. Recent improvements in the technique of contact lens manufacture have now rendered this possible, however, and although only four cases have so far been treated in this manner, we felt that the results were sufficiently good to warrant the issuing of a preliminary report.

Before coming to the clinical aspect of this work it is not out of place to consider the theoretical side. When contact lenses were first produced, it was assumed that a cylindrical correction was unnecessary because astigmatism was entirely due to aberrations in the anterior surface of the cornea, and was therefore neutralised by covering the aspherical surface with a thin layer of saline and a spherical shell of glass. Perhaps this was making 
a virtue of necessity because the type of lens then in vogue had a spherical surface in contact with the bulbar conjunctiva, and theoretically there was therefore nothing to prevent the lens rotating. In practice, however, rotation does not occur, but there is the difficulty that the lens must be inserted in exactly the right position and this would necessitate the making of a fairly conspicuous mark on it which the patient could see. With a contact lens whose scleral surface has been ground so as accurately to fit the ball of the eye, this is not the case, because even if the lens is inserted in a faulty position it tends in a short time to settle down into the position which gives it the closest fit to the eyeball. A good scleral fit, therefore, is of the utmost importance.

The existence of astigmatism which is not due to aberrations in the curvature of the anterior surface of the cornea has been recognised for a long time.

Theoretically, such astigmatism may be due to:-

(1) Aberrations in curvature of the posterior surface of the cornea.

(2) Aberrations in curvature of the anterior and/or posterior surface of the lens.

(3) Radial errors due to the centre of the lens not coinciding with the optical axis of the eye.

(4) Variations in the refractive index of the lens in different meridians.

(5) Tilting of the lens.

It is obvious that none of these can be corrected by a contact lens with spherical corneal surfaces, and it is not surprising therefore that cases arise in which visual acuity is improved by the addition of a cylindrical element to the contact lens.

It is even conceivable that cases which, with ordinary glasses appear to have a spherical error, may when fitted with a contact lens be found to have some residual astigmatism, since it has been stated that most normal eyes show slight corneal astigmatism, but that nature has provided a compensating lenticular defect. If, in such a case, the corneal astigmatism is abolished, the compensating lenticular astigmatism will become manifest, and require correction. The theoretical implications of this are obvious and a considerable amount of work can still be done on the subject, despite the fact that many papers have already been written on the disparity between keratometer readings and the total refraction of the eye.

Turning now to the practical side of the question, a cylindrical correction has not heretofore been ground on a contact lens because it was not possible to combine this with the curvature required on the anterior surface. It has proved possible, however, 
to grind such a correction on the posterior (concave) surface of the lens; but here an interesting complication arises, because we are dealing with the difference between the index of refraction of glass and of the saline which fills the space between the anterior surface of the cornea and the posterior surface of the contact lens.

It follows therefore that, as the difference between the refractive indices of glass and saline is much less than the difference between the refractive indices of glass and air, a more powerful cylinder will be required. Calculation has shown that the increase in strength is approximately 2.5 , i.e., a cylinder of $2.5 \mathrm{D}$. ground on the posterior surface of a contact lens will produce the effect of a 1.0 D. cylinder on the anterior surface. By using this method, satisfactory results have been obtained in the following cases, though it is possible that as the result of further investigations they may be improved upon.

S.G.B. male.-Although this patient's error of refraction was only a small one, he was anxious to have contact lenses. Wearing ordinary spectacles, the right eye with $\frac{\mathrm{sph}-1.0}{\mathrm{cyl}-0.37}$ read $6 / 6$

and the left eye with $\frac{\mathrm{sph}-1.62}{\mathrm{cyl}-0.25} \quad \searrow \quad \operatorname{read} 6 / 6$.

With the usual type of contact lens, each with $\mathrm{sph}+2.50$ ground on it, vision would not improve to beyond $6 / 9 .^{*}$

With the appropriate cylindrical correction ground on to the back of each contact lens, however, each eye could see $6 / 5$. Writing the new contact lens corrections in effective power, they were respectively:-
R.E. $\frac{\mathrm{sph}+2 \cdot 0}{\mathrm{cyl}+1 \cdot 0 \rightarrow 180}$
L.E. $\frac{\mathrm{sph}+2.0}{\mathrm{cyl}+0.75 \rightarrow 180}$

The cylinder, as already explained, was actually 2.5 times this amount. (The same convention is adopted in the following three cases.)

* It should perhaps be explained why a patient with a myopic spectacle correction should require for the rectification of his error of refraction a contact glass with an effective power of $+2.50 \mathrm{D}$. The reason is that in this particular case, the patient's cornea was more curved than the corneal portion of the standard $8 \mathrm{~mm}$. radius contact lens. In consequence of this, without a contact lens, the eye formed an image in front of the retina. When the contact lens was applied, however, the refractive power of the eye was lessened owing to the decreased curvature of its effective anterior surface. In consequence of this, the focus was lengthened and so the image fell behind the retina, thus making the eye hypermetropic and rendering necessary the grinding of a convex lens on to the anterior surface of the contact glass. 
O.G. male.-The next was a moderate myope. The spectacle correction which gave $6 / 5$ partly in each eye was :-

$$
\text { R.E. } \frac{\mathrm{sph}-6.0}{\mathrm{cyl}-0.50} \quad y \quad \text { L.E. } \frac{\mathrm{sph}-5.50}{\mathrm{cyl}-1.0} \leftarrow
$$

With a spherical contact lens $-3.50 \mathrm{D}$ each eye read $6 / 6$, but with a sphero-cylindrical one

$$
\text { R.E. } \frac{\mathrm{sph}-3.0}{\mathrm{cyl}-1.0} \searrow 100 \quad \text { L.E. } \frac{\mathrm{sph}-3.0}{\mathrm{cyl}-1.0} \swarrow_{80}
$$

each eye could read $6 / 5$.

The third patient, M. R. (male), was referred to the Contact Lens Centre by Mr. Myles Bickerton to whom we are indebted for permission to refer to the case. The right eye with spectacle lens saw $6 / 5$, but the left was amblyopic. With a spherical contact lens $+30 \mathrm{D}$ each eye, the right would not improve to beyond $6 / 6$, and the amblyopic left eye saw 6/24. With a sphero-cylindrical contact lens $\frac{\mathrm{sph}+2.75}{\mathrm{cyl}+0.7510} \measuredangle$ the right eye saw $6 / 5$.

The fourth case, Mr. O. aged 19 years, was one of conical cornea. This patient was a West Indian. He developed a small amount of myopia about four years ago and this increased steadily. He was diagnosed in Jamaica as early conical cornea and proceeded to England to obtain contact lenses. With spectacles (concave sphero-cylinders) he could read 6/12 with the right eye, and $6 / 6$ partly with the left, but complained that, although he could see the letters, they had double outlines, which worried him all the time. With a spherical contact lens he read 6/9 R.E. and $6 / 6$ partly L.E. practically the same as with his glasses. By adding a 2.5 cylinder to the back of the contact lens of his left eye, he read $6 / 5$ easily, and all the double outlines disappeared. The right eye was not improved, but he stated that he thought the visual acuity in this eye had always been less than in the left.

\section{ANNOTATION}

\section{The Leeds Reading Aid for Partially Sighted Children}

The Report of the School Medical Officer (Dr. St. Clair Stockwell) for the year ended December 31,1938, in the City of Leeds, contains many items of importance. To ophthalmologists Appendix B, which deals with the subject of our heading, is of great interest. In an historical retrospect we are told that a class for partially blind (now 